\title{
Comparative Evaluation of Dyslipaedemia in Obese and Diabetic Patients of Rural and Urban Areas in Lahore, Pakistan

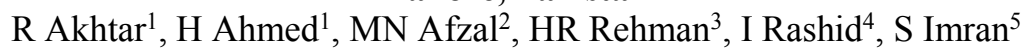

\begin{abstract}
Objective: Obesity and diabetes mellitus are interconnected conditions that share a number of pathophysiological mechanisms such as dyslipidemia leading to cardiovascular complications. The present study was conducted to determine the individual and combined effect of diabetes mellitus and obesity on dyslipidemia and ultimately on silent coronary artery disease (CAD).

Methods: The patients selected from urban and rural areas of Lahore were recruited on the basis of body mass index (BMI) and fasting blood sugar (FBS) and were classified in four groups: (Group A; obese-diabetic, Group B; non-obese and diabetic, Group C; obese and non-diabetic and Group D; non-obese and non-diabetic). Total lipid profile including total cholesterol, triglycerides, high density lipoproteins and low density lipoproteins were determined along with exercise tolerance test (ETT).

Results: The results indicated that combined obesity and diabetes was the major cause of Dyslipaedemia and CAD followed by obesity and diabetes alone. Triglycerides and atherogenic level of HDL-C were more prevalent in obese-diabetic patients (group A) followed by obese (group C) and diabetic (group B) alone. However low density lipoprotein (LDL) was more significant in obese (group C) but the results were also comparable in all other groups.
\end{abstract}

Conclusion: The result of ETT revealed that overall group A was more prone towards CAD as compared to group B and group C but there was a non-significant correlation between CAD and obesity/diabetes within all experimental groups. Moreover, the risk of dyslipidemia and CAD was non significantly higher in urban population than rural population.

Keywords: Coronary artery disease, diabetes mellitus, exercise tolerance test, obesity

From: ${ }^{1}$ Department of Pathology, University of Veterinary and Animal Sciences, Lahore, Pakistan. ${ }^{2}$ Mayo Hospital Lahore, Pakistan.54000. ${ }^{3}$ Department of Physiology, University of Veterinary and Animal Sciences, Lahore, Pakistan. ${ }^{4}$ Department of Parasitology, University of Veterinary and Animal Sciences, Lahore, Pakistan. ${ }^{5}$ Department of Clinical Medicine and Surgery, University of Veterinary and Animal Sciences, Lahore, Pakistan.

Correspondence: Dr R Akhtar, Department of Pathology, University of Veterinary and Animal Sciences, Lahore, Pakistan. E-mail: raheela.akhtar@uvas.edu.pk 


\section{INTRODUCTION}

Dyslipidemia is defined as an elevated total or low-density lipoprotein cholesterol (LDL-C) levels, or low levels of high-density lipoprotein cholesterol (HDL-C) that leads to coronary artery disease (CAD). Type 2 diabetes mellitus and obesity are associated with insulin resistance leading to Dyslipaedemia and finally ending into CAD. Although this chain of pathological events has been studied in past however there is little understanding of percentage involvement of diabetes and obesity in Dyslipaedemia and CAD. The comprehension of role of these two conditions in development of Dyslipaedemia and CAD may be useful for understanding the magnitude of these pathological conditions.

\section{METHODS}

Diabetic patients $(n=160$, with disease duration less than five years) of age 20-65 years and healthy control with mean age $45.42 \pm 10.32$ years were selected from rural and urban areas of Lahore by their informed consent. The patients were divided into four equal groups: Group A; obese-diabetic, Group B; non-obese and diabetic, group C; obese and non-diabetic, group D; non-obese and non-diabetic. Subjects on lipid lowering therapies and with coronary artery disease or diabetic nephropathy were excluded from study. To assess nephropathy, patient's record for complete urine examination and serum creatinine was reviewed. Patients with disabilities impairing to perform exercise tolerance test were also excluded. 


\section{Selection of obese and diabetic patients}

The body mass index was calculated by following relation weight $\mathrm{kg} /$ height $\mathrm{m}^{2}$ and patients were included in respective study groups by their categorization of BMI according to the International Obesity Task Force (1). Patients with healthy weight and BMI of 18.5-24.9 were placed in group B and D. While obese patients having BMI 30 or more were placed in group A and C. Categorization was as followed:

Healthy weight, 18.5-24.9

Overweight, 25.0- 29.9

Class I obesity, 30.0-34.9

Class II obesity, 35- 39.9

Class III obesity, 40.0 or higher

Base line tests were performed for inclusion of diabetic patient which includes fasting blood sugar more than $110 \mathrm{mg} / \mathrm{dL}$ or previously diagnosed diabetes mellitus (2) and further confirmation was performed by glycosylated haemoglobin (HbA1c). For the correlation of obesity and diabetes with asymptomatic coronary artery disease diabetic patients were divided in two groups with $\mathrm{HbAlc}$ level more than $6.5 \%$ as diabetic and with less than $6.5 \%$ as nondiabetic and obese subjects were categorized on the basis of their BMI in five classes and correlation was made by using chi square statistics and results were given with level of significance.

\section{Determination of dyslipedemia}

Dyslipidemia was considered when total cholesterol was more than $200 \mathrm{mg} / \mathrm{dL}$, triglycerides $150 \mathrm{mg} / \mathrm{dL}$ and high density lipoprotein was less than $50 \mathrm{mg} / \mathrm{dL}$ (2). Measurement of total 
cholesterol, triglycerides and high density lipoprotein was conducted by a direct in vitro enzymatic assay using respective Fortress kits. While low density lipoprotein was calculated by using Friedewald formula as described before (3).

\section{Exercise tolerance test}

Asymptomatic coronary artery disease risk was evaluated by exercise tolerance test (ETT). All the included subjects were assessed to rule out contraindication for exercise tolerance test. Modified Bruce protocol was followed for detection of CAD in exercise tolerance test. If ETT was positive then more specific test can be used to rule out false positive.

\section{Determination of correlation of CAD with diabetes and obesity within groups}

In group A and group $\mathrm{C}$ subjects were divided into four sub groups based upon body mass index and then comparison was made allotting 1 for positive and 2 for negative CAD subjects by using Chi square and results were given in level of significance.

\section{Determination of overall correlation of CAD with diabetes and obesity}

For this diabetic patients were divided in two groups with HbA1c level more than $7 \mathrm{mg} / \mathrm{dL}$ as diabetic and with less than $7 \mathrm{mg} / \mathrm{dL}$ as non-diabetic and obese subjects were divided in five categories on the basis of their BMI and correlation was made by using chi square statistic.

\section{Statistical Analysis}

The statistical analysis was performed by employing one way ANOVA and Pearson product moment correlation coefficient using SPSS (version 20.0). Results of ETT were analyzed by using chi square statistic which compares the counts of categorical responses between two or more independent groups. 


\section{RESULTS}

The results of present study indicated that if obesity and diabetes were present simultaneously it increased the risk of dyslipidemia to greater extent. Triglycerides and atherogenic level of HDL$\mathrm{C}$ were more prevalent in obese-diabetic patients (group A) followed by obese and non diabetic patients (group C) and diabetic (group B) alone. Non obese-non diabetic (Group D) had greater HDL-C which is a sign of less atherogenicity. However low density lipoprotein (LDL) was more significant in group $\mathrm{C}$ but the results were also comparable in all other groups (Table 1).

\section{Correlation of HbA1c and different variables}

Correlation between four groups was analyzed by one way of variance and results are given in mean, standard deviation, confidence of interval at $95 \%$ confidence and range within group. This showed group B had worse results mean 8.6850 with standard deviation 1.96019 and greater confidence interval 8.0581-9.3119 at 95\% level of confidence, so the poorer control of blood glucose than the second worse group A. As Group C and D were non-diabetic groups they showed normal HbA1c levels mean 5.4400, SD 0.29071 and 5.7525, 0.43958 respectively.

Correlation of different variables within same group was carried out by Pearson product moment correlation coefficient to obtain additional things for result conclusion and was given with value of " $r$ ". The positive and negative correlations of different variables such as age, BMI and HbAlc with total cholesterol, triglycerides, HDL-C, LDL-C and BMI within the groups are given in Table 2.

Although the results of ETT showed that group A was more prone towards CAD as compared to group $\mathrm{B}$ and $\mathrm{C}$ but this was a non-significant correlation between $\mathrm{CAD}$ and obesity/diabetes within all experimental groups. The linear association between CAD and diabetes/obesity in group A and C was 0.460 and 0.085 and Fisher's exact test in group B was 
0.350. While in control group (group D) just one patient was diagnosed with coronary artery disease and percentage of subjects with risk of CAD was $2.5 \%$ in control subject with no positive risk factor for dyslipidemia. On other hand the overall correlation between CAD and obesity/ diabetes was significant with level of significance 0.048 and 0.010 .

Moreover, the comparative evaluation of asymptomatic CAD in obese and diabetic patients of rural and urban areas of Lahore indicated no significant correlation with $p$ value $(0.102)$.

\section{DISCUSSION}

Diabetes mellitus and obesity are considered as important components of metabolic syndrome. The present study revealed that the co-morbidity of obesity and diabetes mellitus is an important risk factor for Dyslipaedemia leading to high frequency of CAD. This is in agreement with previous findings $(4,5)$ that explained that each component of the metabolic syndrome is an established CAD risk factor and the presence of multiple components confer greater risk than the sum of the risks associated with the individual ones.

The non-significant results of ETT suggested that Dyslipaedemia is an important contributor to CAD in insulin-resistant conditions such as diabetes mellitus and obesity.

Our results revealed that obesity was a stronger factor for hypercholesterolemia than diabetes in understudy subjects. These results are different from past studied and this could be due to effect of medications as diabetic patients were on oral hypoglycemic agents or on insulin therapy, and taking healthy foods. Our study revealed that the triglyceride level has weak negative correlation with glycaemic control in obese-diabetic (group A) and diabetic ( group B) which is contrary to previous work (6) that explained positive correlation between blood glucose and triglycerides. 
These results could be implicated in future for controlling the complications of diabetes mellitus and obesity in terms of coronary artery disease.

\section{CONCLUSION}

The results of present study indicated that if obesity and diabetes were present simultaneously it increased the risk of dyslipidemia to greater extent. However, there was a non-significant relation between Dyslipaedemia and CAD. Moreover, the risk of dyslipidemia and CAD was non significantly higher in urban population than rural population. 


\section{REFERENCES}

1. Geneva. Obesity: preventing and managing the global epidemic: report of a WHO Consultation on Obesity. World Health Organization. 1998.

2. Harold E, Bays RH, Chapman KM, Fox, Susan G. Comparison of self-reported survey (SHIELD) versus NHANES data in estimating prevalence of dyslipidemia. Curr Med Res Opin 2008; 24: 1179-86.

3. Kapoor R, Montosh CM, Singh N. A leap above Friedewald formula for calculation of low-density lipoprotein-cholesterol. J Lab Physicians 2015; 7: 11-6.

4. Dixon JB, O'Brien PE. Lipid profile in the severely obese, changes with weight loss after lap-band surgery. Obes Res 2002; 10: 903-10.

5. Victor HB, Korita I, Bulo A. How is metabolic syndrome related to dyslipidemia? Biochem Med 2008; 18: 14-24.

6. Khan PM, Humayun M, Khan GA. Lipid profile in uncontrolled diabetes mellitus. J Post Med Ins 1998; 12: 81-3. 
Table-1: Comparison of Lipid Profile between different groups.

\begin{tabular}{llllll}
\hline & & Group=A & Group=B & Group=C & Group=D \\
Total & Mean (SD) & 182.35 & $178.3(29.8)$ & 190.5 & 158.1 \\
Cholesterol & & $(34.5)$ & & $(39.63)$ & $(28.85)$ \\
& $95 \%$ C I for & $171.3264-$ & $168.7916-$ & $177.8492-$ & $148.8718-$ \\
& Mean & 193.3736 & 187.8584 & 203.2008 & 167.3282 \\
Triglycerides & Range & $95.0-246.0$ & $121.0-253.0$ & $150.0-314.0$ & $95.0-223.0$ \\
& Mean (SD) & 191.0 & 140.7 & 156.75 & 125.02 \\
& & $(85.92)$ & $(54.78)$ & $(68.2)$ & $(19.4)$ \\
& $95 \%$ C I for & $163.5196-$ & $123.1791-$ & $134.9407-$ & $118.8077-$ \\
HDL-C & Mean & 218.4804 & 158.2209 & 178.5593 & 131.2423 \\
& Range & $74.0-403.0$ & $49.0-287.0$ & $73.0-306.0$ & $87.0-178.0$ \\
& Mean (SD) & 43.27 & $45.92(8.45)$ & $44.7(8.22)$ & $54.22(6.04)$ \\
& & $(16.54)$ & & & \\
LDL-C & 95\% C I for & $37.9831-$ & $43.2206-$ & $42.0695-$ & $52.2932-$ \\
& Mean & 48.5669 & 48.6294 & 47.3305 & 56.1568 \\
& Range & $18.0-123.0$ & $31.0-77.0$ & $30.0-67.0$ & $42.0-65.0$ \\
& Mean (SD) & 115.67 & 112.15 & 120.62 & 111.17 \\
& & $(35.3)$ & $(30.2)$ & $(31.5)$ & $(19.3)$ \\
& $95 \%$ C I for & $104.3854-$ & $102.4808-$ & $110.5529-$ & $104.9960-$ \\
& Mean & 126.9646 & 121.8192 & 130.6971 & 117.3540 \\
& Range & $28.0-216.0$ & $25.0-174.0$ & $84.0-201.0$ & $67.0-156.0$ \\
\hline
\end{tabular}

Group B: Non-Obese and Diabetic Group A: Obese and Diabetic. Group C: Obese and Non-Diabetic Group D: Non-Obese and NonDiabetic, Total- $\mathrm{C}=$ total cholesterol, $\mathrm{BMI}=$ body mass index, $\mathrm{HbAl}=$ glycosylated haemoglobin, HDL-C $=$ high density lipoprotein cholesterol, LDL-C = low density lipoprotein cholesterol. 
Table 2: Correlation of different variables within experimental Groups

\begin{tabular}{|c|c|c|c|c|c|c|c|c|c|c|c|c|}
\hline & \multirow[b]{2}{*}{ Age } & \multicolumn{2}{|c|}{ Group A } & \multicolumn{3}{|c|}{ Group B } & \multicolumn{3}{|c|}{ Group C } & \multicolumn{2}{|c|}{ Group D } & \multirow[b]{2}{*}{$\mathrm{HbA1c}$} \\
\hline & & BMI & HbA1c & Age & BMI & HbAlc & Age & BMI & HbA1c & Age & BMI & \\
\hline $\begin{array}{l}\text { Total } \\
\text { Cholesterol }\end{array}$ & 0.128181 & -0.18805 & -0.14657 & 0.101336 & -0.05019 & -0.14657 & 0.018907 & 0.284077 & 0.101709 & -0.05663 & 0.267317 & 0.001956 \\
\hline Triglyceride & -0.11533 & -0.09293 & -0.089 & 0.177373 & 0.048585 & -0.089 & 0.195359 & 0.299101 & 0.344201 & 0.049986 & -0.07476 & 0.441273 \\
\hline HDL-C & 0.293495 & -0.27062 & 0.157768 & -0.14194 & 0.072597 & 0.157768 & -0.30506 & -0.22997 & -0.14978 & 0.180356 & -0.05917 & 0.04877 \\
\hline LDL-C & 0.116593 & -0.14146 & -0.16025 & 0.10549 & -0.21428 & -0.16025 & -0.06778 & 0.104236 & 0.225501 & -0.00755 & 0.464108 & 0.107373 \\
\hline BMI & -0.17898 & & -0.13277 & 0.006341 & & 0.006341 & 0.232329 & & 0.07696 & 0.034304 & & 0.087079 \\
\hline
\end{tabular}

